\title{
Padrões do sono na população pantaneira
}

\author{
Sleep patterns in the pantaneira population \\ Patrones de sueño en la población pantaneira
}

Recebido: 21/02/2021 | Revisado: 01/03/2021 | Aceito: 04/03/2021 | Publicado: 11/03/2021

Carlos Eduardo Vilela Gaudioso

ORCID: https://orcid.org/0000-0001-5642-4302 Universidade Federal de Mato Grosso do Sul, Brasil E-mail: carlos.gaudioso@ufms.br

Luis Alberto Magna

ORCID: https://orcid.org/0000-0001-8113-6986 Universidade Estadual de Campinas, Brasil E-mail: lamagna@uol.com.br

José Carlos Souza

ORCID: https://orcid.org/0000-0003-4460-3770 Universidade Estadual de Mato Grosso do Sul, Brasil E-mail: josecarlossouza@uol.com.br Daniela Vicimansa Mônaco

ORCID: https://orcid.org/0000-0001-7581-7734 Universidade Estadual de Campinas, Brasil E-mail: danielamonacoferreira@gmail.com

\begin{abstract}
Resumo
Introdução: o sono é vital, caracterizado como um conjunto de alterações comportamentais e fisiológicas. A pesquisa foi realizada numa população rural, isolada, pantaneira, residente na maior planície alagada do planeta. Objetivos: Identificar os padrões do sono na população pantaneira. Método: Estudo quantitativo, descritivo, analítico, de corte transversal e comparativo, o total da amostra foi de 789 participantes, distribuídos em população rural (25\%) e urbana (75\%). Para a análise foi utilizado métodos de estatística descritiva, com estimativa de proporções e correspondente erro-padrão da média. A análise univariada consistiu em comparação de proporções pelo teste qui-quadrado e comparação de médias. $\mathrm{O}$ ajuste à distribuição normal foi aferido por teste não paramétrico. Resultados: Constatou-se aumento significativo da proporção de alteração do sono com o aumento da faixa etária, diminuindo com o aumento do nível de escolaridade. As alterações do sono ocorreram em maior proporção entre as mulheres, entre aquelas que têm escolaridade até o ensino médio, que possuem maior número de filhos. Conclusão: A divulgação destes achados é essencial para a promoção da saúde integral do pantaneiro, visando superar problemas relacionados ao sono e à qualidade de vida.
\end{abstract}

Palavras-chave: Sono; População rural; Áreas alagadas.

\begin{abstract}
Introduction: sleep is vital, characterized as a set of behavioral and physiological changes. The research was carried out in a rural, isolated, Pantanal population, residing in the largest floodplain on the planet. Objectives: To identify sleep patterns in the Pantanal population. Method: Quantitative, descriptive, analytical, cross-sectional and comparative study, the total sample was 789 participants, distributed in rural (25\%) and urban (75\%) populations. For the analysis, descriptive statistical methods were used, with proportions estimation and corresponding standard error of the mean. The univariate analysis consisted of comparing proportions using the chi-square test and comparing means. Adjustment to normal distribution was assessed using a non-parametric test. Results: There was a significant increase in the proportion of sleep disorders with increasing age, decreasing with increasing level of education. Sleep changes occurred in a greater proportion among women, among those who have schooling up to high school, who have a greater number of children. Conclusion: The dissemination of these findings is essential for the promotion of the integral health of the Pantanal, aiming to overcome problems related to sleep and quality of life.
\end{abstract}

Keywords: Sleep; Rural population; Flooded areas.

\section{Resumen}

Introducción: el sueño es vital, caracterizado por un conjunto de cambios conductuales y fisiológicos. La investigación se llevó a cabo en una población rural y aislada del Pantanal, que reside en la llanura aluvial más grande del planeta. Objetivos: Identificar patrones de sueño en la población del Pantanal. Método: Estudio cuantitativo, descriptivo, analítico, transversal y comparativo, la muestra total fue de 789 participantes, distribuidos en población rural $(25 \%)$ y urbana $(75 \%)$. Para el análisis se utilizaron métodos estadísticos descriptivos, con estimación de proporciones y correspondiente error estándar de la media. El análisis univariado consistió en comparar proporciones 
mediante la prueba de chi-cuadrado y comparar medias. El ajuste a la distribución normal se evaluó mediante una prueba no paramétrica. Resultados: Hubo un aumento significativo en la proporción de trastornos del sueño con el aumento de la edad, disminuyendo con el aumento del nivel de educación. Los cambios de sueño ocurrieron en mayor proporción entre las mujeres, entre las que tienen escolaridad hasta el bachillerato, que tienen mayor número de hijos. Conclusión: La difusión de estos hallazgos es fundamental para la promoción de la salud integral del Pantanal, con el objetivo de superar los problemas relacionados con el sueño y la calidad de vida.

Palabras clave: Sueño; Población rural; Humedales.

\section{Introdução}

O sono é caracterizado como um fenômeno de vital importância para o ser humano agregando funções de proteção, restauração e conservação de energia, sendo que a sua ausência denota deficiências nas atividades diárias básicas do ser humano, interações sociais, psicológicas ou cognitivas (Neves et al., 2013). O ato de dormir possui funções biológicas fundamentais englobando a consolidação da memória, termorregulação, renovo e conservação de energia e homeostase das funções endócrinas (Ribeiro et al., 2014). Assim, é um processo biológico essencial para o ser humano, pois mantém o equilíbrio da vida. A sua quantidade e a qualidade são de extrema importância, visto que qualquer alteração pode interferir no dia a dia e na saúde do indivíduo (Godnho et al., 2017).

Os seres humanos possuem hábitos especificamente diurnos possuindo sua atividade e vigília concentradas durante o dia, e o sono à noite, sendo que, um tempo reduzido de sono pode influenciar negativamente nas atividades diárias corroborando para riscos de acidentes e baixo rendimento (Ferreira \& Martino, 2012), basicamente dois processos regulam o sono, o Homeostático que buscará o equilíbrio entre a necessidade de descanso quando a vigília é alongada, e o Circadiano o qual é controlado por um marca-passo endógeno independente do sono e da vigília (Soares \& Almondes, 2012).

Os transtornos do sono podem gerar vários problemas e prejuízos fisiológicos e psicológicos, já que o sono desempenha uma ação crucial na homeostase do organismo. Os distúrbios relacionados a essa necessidade fisiológica podem desempenhar um papel na associação entre estresse no trabalho e saúde cardiovascular (Ghiciuc et al., 2016). Os prejuízos causados podem gerar déficit cognitivo, notável dificuldade de concentração e irritabilidade. Então, é importante destacar que os efeitos e prejuízos desencadeados por distúrbios do sono não se restringem ao período noturno, mas podem exercer influências ao longo do dia e na QV dos indivíduos afetados (Oliveira et al., 2019).

A National Sleep Foundation alerta sobre a má higiene do sono (irregularidade do início do sono entre os dias da semana e fins de semana, acesso a mídias eletrônicas na proximidade com o horário de dormir, uso de substâncias excitantes lícitas ou ilícitas ou consumo de bebidas cafeinadas) e propõe a aplicação de uma rotina diária de higiene adequada do sono. Portanto, é necessária uma investigação mais abrangente dos aspectos e transtornos do sono por profissionais de saúde para poder gerar uma melhoria na expectativa de vida e na saúde dos indivíduos (Oliveira et al., 2019).

Reduzir a obesidade e o sedentarismo através de métodos para desenvolver comportamentos saudáveis pode ser um caminho a ser traçado para minimizar algumas complicações geradas pelos distúrbios do sono, entre as estratégias estão projetos de atividade física, campanhas educativas e dieta de baixo conteúdo de gordura. Também podem trazer benefícios incluir campanhas de conscientização do sono. A Associação Brasileira do Sono promove anualmente a Semana do Sono, a proposta é alcançar a população geral com temas e assuntos de relevância em todas as regiões do Brasil por meio dos seus associados, promove ainda, o Congresso Brasileiro do Sono, um evento científico de atualização e difusão das descobertas científicas no assunto (Associação Brasileira do Sono, 2019).

Pesquisar os hábitos e distúrbios do sono é um princípio importante para trabalhos futuros no que concerne à qualidade e hábitos saudáveis da população pantaneira, em decorrência de suas peculiaridades. No Pantanal Sul-matogrossense, o acesso é difícil, a cultura local é caracterizada pelos hábitos conservadores das pessoas que vivem nessa região, temerosos com a urbanização, adultos pouco escolarizados, sensação ambivalente da necessidade dos recursos que a cidade 
oferece, versus a calmaria e o isolamento.

O Estado do Mato Grosso do Sul abriga 62\% da bacia pantaneira, a maior planície inundável do planeta, declarada Patrimônio Nacional pela Constituição Federal Brasileira de 1988, localizada no centro da América do Sul, pertencendo ao Paraguai, Bolívia e Brasil, destes 6\% distribuídos nos países estrangeiros e 32\% no Estado do Mato Grosso, considerado ainda Patrimônio Natural Mundial pela Organização das Nações Unidas para a Educação, a Ciência e a Cultura e Reserva da Biosfera (Mioto et al., 2012; Harris et al., 2005).

O período de inundação, também chamado ciclo das águas, é o fenômeno ecológico mais relevante do Pantanal recebendo do Rio Paraguai e afluentes, transformando o cenário da região através da biodiversidade, sendo "interseção de quatro grandes Regiões Fitoecológicas, que regionalmente podem ser conhecidas como Mata Decídua, Mata Semidecídua, Cerrado e Chaco" (Abdon et al., 2007).

Considerado um dos poucos municípios pantaneiros, Coxim em sua congruência entre os rios Coxim e Taquari que fica à montante da ponte sobre a BR 163 , no Km 727, passa a ser denominado de médio Taquari, caracterizado por suas águas mais tranquilas, já em transição de depressão para planície, formando bancos de areia, ilhas, baías entre outros fatores que caracterizam um rio pantaneiro e por isso, Coxim é considerado como o Portal do Pantanal (Coxim, 2015).

Todo o território coxinense que se encontra fora da planície pantaneira é considerada como influente corredor ecológico migratório de abastecimento e intercâmbio de biodiversidades entre o Pantanal e o Cerrado (Coxim, 2015).

A cidade de Coxim é um centro econômico e regional, sendo nacionalmente conhecida como Capital do Peixe e Terra do Pé de Cedro, em decorrência da canção de Zacarias Mourão. Abriga diversos ícones paisagísticos, como as cachoeiras do Salto, das Palmeiras e os Rios Taquari e Coxim. Sua economia baseia-se na agropecuária (Coxim, 2015).

O homem pantaneiro é o indivíduo nascido no Pantanal ou aquele que, mesmo não tendo nascido na região, adotou o modo de vida, partilhando dos usos e dos costumes próprios dessa população (Nogueira, 1990).

O Pantanal e o pantaneiro se completam, se interagem, e um precisa do outro para sobreviver. Só o verdadeiro peão, que conhece e desbrava todos os dias o Pantanal, é conhecedor da natureza local: ele sabe tudo daquele lugar, ele tem suas particularidades, desde a sua crença e vida, é ele quem decifra o Pantanal, a vestimenta de campeiro, sua fala, marcada de neologismos e coloquialismos, suas festas, o tereré, bebida típica da região e tantas outras características marcantes compõem o extenso universo do homem pantaneiro (Delamo et al., 2010).

As influências das inúmeras culturas dos diversos cantos do país e das fronteiras de países vizinhos oferecem uma mistura farta para o aparecimento do homem pantaneiro, bandeirantes, nordestinos, cafusos, caboclos, mulatos e os paraguaios e bolivianos, que adentraram o território brasileiro em busca de trabalho. Toda essa influência de etnias, usos e costumes legou ao pantaneiro modos de vida que foram se modificando ao longo dos anos (Finocchio, 1998).

A principal atividade desse homem pantaneiro é a pecuária, possui seus costumes e hábitos específicos, teve que se adaptar ao clima, alimentação e onde dormir, é costume do pantaneiro dormir cedo e acordar ainda pela madrugada, sua primeira refeição é o quebra-torto, arroz com carne seca e café com leite. De três a quatro vezes ao dia, em horários préestabelecidos, interrompe o seu trabalho para tomar o seu tereré (bebida de origem paraguaia, usado com erva mate e água gelada ou até mesmo retirada dos corixos). Uma outra característica do peão pantaneiro é a contemplação da natureza, através disso ele descobre alterações do tempo, mudança e influência da lua, indícios de chuva, enchentes ou estiagens prolongadas. Tudo isso representa um pouco de sua sabedoria.

\section{Casuística e Método}

Trata-se de um estudo quantitativo, descritivo, analítico, de corte transversal e comparativo, no qual foi realizado um pré-teste com amostra aleatória para adaptação dos termos existentes nos formulários e previsão de tempo de respostas. 
Para este trabalho, foram utilizadas como referência as indicações de Pereira AS et al (2018), para estudos e trabalhos com essas características metodológicas.

Este estudo é parte integrante da tese de doutorado da Faculdade de Ciências Médicas da Universidade Estadual de Campinas, onde sua abrangência deriva do sono e qualidade de vida da população pantaneira, os dados sócio demográficos já foram extraídos e pode ser consultado através da publicação intitulada "Perfil sociodemográfico da População Pantaneira do Paiaguás", podendo ser acessado através do link: https://rsdjournal.org/index.php/rsd/article/view/7123/6282.

O total da amostra foi de 789 participantes, distribuídos em população rural e urbana, em uma proporção de $25 \%$ para rural e 75\% urbana. O período de coleta de dados foi de setembro de 2017 a outubro de 2018, convergiu a diminuição dos alagamentos e das chuvas, chamado também ciclo das águas do pantanal, período em que a estiagem é característica e que oferece acesso a bacia pantaneira, devido aos fenômenos naturais, compreendendo os meses de agosto a novembro.

Foram aplicados os seguintes instrumentos para estudo e análise: a) Pittsburgh Sleep Quality Index (PSQI); Questionário de Berlim (QB, Berlin Questionnaire); e o Questionário Sociodemográfico com as seguintes variáveis independentes; idade, sexo, religião, naturalidade, escolaridade, há quanto tempo reside na região, ocupação e há quanto tempo está nesta ocupação, renda, jornada diária em horas de trabalho.

As variáveis dependentes foram as identificadas pela aplicação dos instrumentos: Questionário de Berlim (QB, Berlin Questionnaire), a versão de 1999, validada por Nikolaus C. Netzer et al., instrumento de avaliação da patologia respiratória do sono, foi adaptada e traduzida para o português por A. P. Vaz et al., em 2011, que identifica alteração do sono (não ou ausente, e sim ou presente) (Anexo B).

O QB é um instrumento desenvolvido por especialistas em apneia do sono reunidos em Berlim, Alemanha, em 1996, de auto relato, tem sido comumente usado como medida de triagem para a apneia do sono, especialmente ao longo dos últimos anos. Avalia a presença e frequência de comportamento do ronco, sonolência ou cansaço ao acordar e história de obesidade ou hipertensão, composto por 9 questões breves mais o índice de massa corporal.

Para os itens nas categorias 1 e 2, é atribuído 1 ponto na presença ou ocorrência de um sintoma de forma persistente ou frequente (3-4 vezes por semana). O item 5, referente às apneias presenciadas, é uma exceção, sendo que para os mesmos pressupostos são atribuídos 2 pontos. A categoria 2 apresenta um item adicional referente à frequência com que ocorre sonolência (item 9), para a qual não está registrada nenhuma pontuação. As categorias 1 e 2 são positivas quando a soma da pontuação de todos os itens é igual ou superior a 2, e a categoria 3, na presença de hipertensão arterial e/ou obesidade. Registro positivo em duas ou três categorias define um score de alto risco para a Síndrome da Apneia Obstrutiva do Sono, e o registro positivo em apenas uma categoria ou nenhuma define um score de baixo risco (Vaz et al., 2011).

Como variável dependente incluiu também o PSQI, que afere a qualidade do sono (boa ou ruim), a versão do PSQI (1989), desenvolvida por Daniel J. Buysse et al., instrumento de avaliação da qualidade do sono, foi adaptada e traduzida para o português, considerado a cultura da população brasileira e traduzido por A. N. Bertolazi, em 2008.

Com o intuito de fornecer uma medida de qualidade de sono uniformizada, o PSQI é fácil de ser respondido e interpretado, faz a distinção entre "bons dormidores" e "maus dormidores".

O questionário consiste de 19 questões auto administradas e 5 questões respondidas por seus companheiros de quarto. Estas últimas são utilizadas somente para informação clínica e não foram utilizadas nesta pesquisa. As 19 questões são agrupadas em 7 componentes, com pesos distribuídos numa escala de 0 a 3. Estes itens do PSQI, seguem padrões de características rotineiramente avaliadas em entrevistas clínicas de pacientes com queixas em relação ao sono, são a qualidade subjetiva do sono, a latência para o sono, a duração do sono, a eficiência habitual do sono, os transtornos do sono, o uso de medicamentos para dormir e a disfunção diurna. As pontuações destes componentes são então somadas para produzirem um escore global, que varia de 0 a 21, onde, quanto maior a pontuação, pior a qualidade do sono. Um escore global do PSQI>5 
indica que o indivíduo está apresentando grandes dificuldades em pelo menos 2 componentes, ou dificuldades moderadas em mais de 3 componentes (Bertolazi, 2008).

A população estudada é a residente no pantanal do Paiaguás, agrupando grande parte da população do município de Coxim, MS, (32.159 habitantes), da qual foi obtida uma amostra de 789 indivíduos acima de 15 anos, estratificados por sexo e faixa etária. Todos os dados foram inseridos em uma planilha do programa Excel $2013^{\circledR}$, e transferidos para o programa estatístico EBM-SPSS v. 21.

A estimativa do tamanho da amostra considerou margem de erro máxima, para as variáveis dependentes, de 5\% para mais ou para menos, e ainda $\alpha=5 \%$ e poder de $80 \%$ (Instituto Brasileiro de Geografia e Estatística[IBGE], 2019).

As variáveis dependentes, ambas de distribuição binomial, foram as identificadas pela aplicação dos instrumentos QB, que identifica alteração do sono (não ou ausente, e sim ou presente); e PSQI, que afere a qualidade do sono (boa ou ruim).

Foram utilizados métodos de estatística descritiva, com a estimativa de proporções (variáveis de atributos e categóricas) e correspondente erro padrão da média, e média e erro padrão da média das variáveis quantitativas.

A análise univariada foi feita pela comparação de proporções pelo teste do Qui-quadrado, e comparação de médias (teste $t$ de Student, após a verificação da homogeneidade das variâncias pelo teste de Levene, para variáveis com distribuição normal; e teste não paramétrico de Mann-Whitney para variáveis sem distribuição normal). $\mathrm{O}$ ajuste à distribuição normal foi aferido pelo teste de Kolmogorov-Smirnov.

As variáveis independentes que, na análise univariada, mostraram associação significativa com as variáveis dependentes, foram incluídas em modelo de análise de regressão linear múltipla escalonada (stepwise forward, multiple linear regressions) com cada uma delas.

Em todos os casos foi adotado o nível de $5 \%(p<0,05)$ para a declaração de existência de significância no teste aplicado.

A Tabela 1 apresenta os resultados da análise univariada do resultado do QB alteração do sono) em função das variáveis independentes sociodemográficas. Por concisão, o resultado do QB será referenciado pelo acrônimo QB e quando positivo, o que é identificado pela detecção de alteração do sono, anotado como sim.

Tabela 1. Análise univariada do resultado do Questionário Berlim em função das variáveis independentes sociodemográficas.

\begin{tabular}{|c|c|c|c|c|c|c|c|}
\hline \multirow{3}{*}{ Variável } & \multicolumn{6}{|c|}{ QB - alteração do sono } & \multirow{3}{*}{$p$} \\
\hline & \multicolumn{2}{|c|}{ Não } & \multicolumn{2}{|c|}{ Sim } & \multicolumn{2}{|c|}{ Total } & \\
\hline & $\mathbf{N}$ & $\%$ & $\mathbf{n}$ & $\%$ & $\mathbf{n}$ & $\%$ & \\
\hline \multicolumn{8}{|l|}{ Sexo: } \\
\hline Feminino & 215 & 61,1 & 137 & 38,9 & 352 & 100,0 & 0,892 \\
\hline Masculino & 202 & 61,6 & 126 & 38,4 & 328 & 100,0 & \\
\hline \multicolumn{8}{|l|}{ Faixa etária (anos): } \\
\hline 15 a 19 & 107 & 70,4 & 45 & 29,6 & 152 & 100,0 & \multirow{7}{*}{$<0,001$} \\
\hline 20 a 29 & 143 & 69,1 & 64 & 30,9 & 207 & 100,0 & \\
\hline 30 a 39 & 68 & 58,6 & 48 & 41,4 & 116 & 100,0 & \\
\hline 40 a 49 & 55 & 56,7 & 42 & 43,3 & 97 & 100,0 & \\
\hline 50 a 59 & 22 & 37,9 & 36 & 62,1 & 58 & 100,0 & \\
\hline 60 a 69 & 12 & 44,4 & 15 & 55,6 & 27 & 100,0 & \\
\hline 70 ou mais & 6 & 35,3 & 11 & 64,7 & 17 & 100,0 & \\
\hline \multicolumn{8}{|l|}{ Escolaridade: } \\
\hline Sem & 25 & 43,9 & 32 & 56,1 & 57 & 100,0 & \multirow{3}{*}{0,005} \\
\hline Até Ensino Fundamental 2 & 120 & 56,9 & 91 & 43,1 & 211 & 100,0 & \\
\hline Até Ensino Médio & 187 & 65,6 & 98 & 34,4 & 285 & 100,0 & \\
\hline
\end{tabular}




\begin{tabular}{|c|c|c|c|c|c|c|c|}
\hline \multirow{3}{*}{ Variável } & \multicolumn{6}{|c|}{ QB - alteração do sono } & \multirow{3}{*}{$p$} \\
\hline & \multicolumn{2}{|c|}{ Não } & \multicolumn{2}{|c|}{ Sim } & \multicolumn{2}{|c|}{ Total } & \\
\hline & $\mathbf{N}$ & $\%$ & $\mathbf{n}$ & $\%$ & $\mathbf{n}$ & $\%$ & \\
\hline Superior até pós-graduação & 77 & 67,0 & 38 & 33,0 & 115 & 100,0 & \\
\hline \multicolumn{8}{|l|}{ Cor: } \\
\hline Branca & 116 & 54,7 & 96 & 45,3 & 212 & 100,0 & \multirow{4}{*}{0,002} \\
\hline Parda & 214 & 60,6 & 139 & 39,4 & 353 & 100,0 & \\
\hline Negra & 54 & 67,5 & 26 & 32,5 & 80 & 100,0 & \\
\hline Amarela & 23 & 92,0 & 2 & 8,0 & 25 & 100,0 & \\
\hline \multicolumn{8}{|l|}{ Estado civil: } \\
\hline Solteiro & 224 & 65,9 & 116 & 34,1 & 340 & 100,0 & \multirow{4}{*}{0,069} \\
\hline Casado ou união estável & 163 & 58,0 & 118 & 42,0 & 281 & 100,0 & \\
\hline Separado ou divorciado & 20 & 48,8 & 21 & 51,2 & 41 & 100,0 & \\
\hline Viúvo & 8 & 57,1 & 6 & 42,9 & 14 & 100,0 & \\
\hline \multicolumn{8}{|l|}{ Filhos: } \\
\hline Não & 198 & 66,7 & 99 & 33,3 & 297 & 100,0 & \multirow[t]{2}{*}{0,012} \\
\hline Sim & 218 & 57,2 & 163 & 42,8 & 381 & 100,0 & \\
\hline \multicolumn{8}{|l|}{ Reside com: } \\
\hline Sozinho & 38 & 58,5 & 27 & 41,5 & 65 & 100,0 & \multirow{3}{*}{0,540} \\
\hline Família & 363 & 61,9 & 223 & 38,1 & 586 & 100,0 & \\
\hline Outro & 13 & 52,0 & 12 & 48,0 & 25 & 100,0 & \\
\hline \multicolumn{8}{|l|}{ Moradia: } \\
\hline Própria & 288 & 59,6 & 195 & 40,4 & 483 & 100,0 & \multirow{4}{*}{0,587} \\
\hline Alugada & 64 & 62,1 & 39 & 37,9 & 103 & 100,0 & \\
\hline Cedida & 38 & 63,3 & 22 & 36,7 & 60 & 100,0 & \\
\hline Outro & 10 & 76,9 & 3 & 23,1 & 13 & 100,0 & \\
\hline \multicolumn{8}{|l|}{ Aposentado: } \\
\hline Não & 391 & 62,5 & 235 & 37,5 & 626 & 100,0 & \multirow[t]{2}{*}{0,066} \\
\hline Sim & 23 & 48,9 & 24 & 51,1 & 47 & 100,0 & \\
\hline \multicolumn{8}{|l|}{ Religião: } \\
\hline Católica & 216 & 61,5 & 135 & 38,5 & 351 & 100,0 & \multirow{4}{*}{0,764} \\
\hline Evangélica & 160 & 62,0 & 98 & 38,0 & 258 & 100,0 & \\
\hline Espírita & 13 & 72,2 & 5 & 27,8 & 18 & 100,0 & \\
\hline Outra & 23 & 57,5 & 17 & 42,5 & 40 & 100,0 & \\
\hline
\end{tabular}

Legenda: QB - Questionário de Berlim.

Fonte: Gaudioso et al. (2020).

Observa-se um aumento significativo da proporção de alteração do sono com o aumento da faixa etária, o que é também corroborado pela comparação das distribuições da idade segundo QB pelo teste não paramétrico de Mann-Whitney, com a média de idade no grupo sem alteração ficando em 30,1 anos e em 36,7 anos no grupo com alteração ( $p<0,001)$. Dá-se o contrário quanto ao efeito do nível de escolaridade, isto é, diminui a proporção de alteração do sono com o aumento do nível de escolaridade.

Em relação à cor declarada pelo entrevistado, observa-se uma pequena proporção entre os identificados pela cor amarela, ficando os demais grupos em proporção semelhante $(p=0,115)$. Finalmente, ter filhos aumenta a proporção de alteração do sono revelada pelo instrumento QB.

Cabe comentar que tanto o estado civil quanto a inatividade (aposentado), muito embora não atinjam o nível de significância adotado, situam-se num intervalo de valor de $p$ que aponta uma possível tendência.

$\mathrm{Na}$ Tabela 2 estão os resultados da análise univariada do QB em função das variáveis independentes sociodemográficas complementares. 
Tabela 2. Análise univariada do resultado do Questionário Berlim em função das variáveis independentes sociodemográficas complementares.

\begin{tabular}{|c|c|c|c|c|c|c|c|}
\hline \multirow{3}{*}{ Variável } & \multicolumn{6}{|c|}{ QB - alteração do sono } & \multirow{3}{*}{$p$} \\
\hline & \multicolumn{2}{|c|}{ Não } & \multicolumn{2}{|c|}{ Sim } & \multicolumn{2}{|c|}{ Total } & \\
\hline & $\mathbf{N}$ & $\%$ & n & $\%$ & $\mathbf{n}$ & $\%$ & \\
\hline \multicolumn{8}{|c|}{ Número de filhos: } \\
\hline 1 & 84 & 68,9 & 38 & 31,1 & 122 & 100,0 & \multirow{3}{*}{0,003} \\
\hline 2 & 70 & 55,6 & 56 & 44,4 & 126 & 100,0 & \\
\hline 3 ou mais & 54 & 47,0 & 61 & 53,0 & 115 & 100,0 & \\
\hline \multicolumn{8}{|c|}{ Horas de trabalho: } \\
\hline Menos de 8 & 70 & 56,0 & 55 & 44,0 & 125 & 100,0 & \multirow{3}{*}{0,689} \\
\hline 8 & 143 & 60,6 & 93 & 39,4 & 236 & 100,0 & \\
\hline Mais de 8 & 51 & 58,0 & 37 & 42,0 & 88 & 100,0 & \\
\hline \multicolumn{8}{|c|}{ Renda pessoal (salário mínimo): } \\
\hline Menos de 1 & 104 & 60,1 & 69 & 39,9 & 173 & 100,0 & \multirow{3}{*}{0,906} \\
\hline Entre 1 e 2 & 121 & 59,0 & 84 & 41,0 & 205 & 100,0 & \\
\hline Mais de 2 & 72 & 61,5 & 45 & 38,5 & 117 & 100,0 & \\
\hline \multicolumn{8}{|c|}{ Renda familiar (salário mínimo): } \\
\hline Menos de 1 & 49 & 65,3 & 26 & 34,7 & 75 & 100,0 & \multirow{3}{*}{0,819} \\
\hline Entre 1 e 2 & 103 & 61,3 & 65 & 38,7 & 168 & 100,0 & \\
\hline Mais de 2 & 190 & 61,7 & 118 & 38,3 & 308 & 100,0 & \\
\hline \multicolumn{8}{|c|}{ Tempo de residência (anos): } \\
\hline Menos de 10 & 88 & 65,2 & 47 & 34,8 & 135 & 100,0 & \multirow{4}{*}{0,267} \\
\hline 10 a 20 & 103 & 62,8 & 61 & 37,2 & 164 & 100,0 & \\
\hline 20 a 30 & 100 & 62,5 & 60 & 37,5 & 160 & 100,0 & \\
\hline 30 ou mais & 115 & 55,6 & 92 & 44,4 & 207 & 100,0 & \\
\hline
\end{tabular}

Legenda: QB - Questionário de Berlim.

Fonte: Gaudioso et al. (2020).

Na Tabela 3 é mostrada a análise univariada de QB em função de alguns hábitos pertinentes à população estudada. Nesse sentido, verificou-se que o hábito da ingestão de café está associado a um aumento da proporção de alteração do sono. 
Tabela 3. Análise univariada do resultado Questionário Berlim em função de alguns hábitos da população estudada.

\begin{tabular}{|c|c|c|c|c|c|c|c|}
\hline \multirow{3}{*}{ Variável } & \multicolumn{6}{|c|}{ QB - alteração do sono } & \multirow{3}{*}{$p$} \\
\hline & \multicolumn{2}{|c|}{ Não } & \multicolumn{2}{|c|}{ Sim } & \multicolumn{2}{|c|}{ Total } & \\
\hline & $\mathbf{n}$ & $\%$ & $\mathbf{n}$ & $\%$ & n & $\%$ & \\
\hline \multicolumn{8}{|c|}{ Bebida alcoólica: } \\
\hline Não & 333 & 62,8 & 197 & 37,2 & 530 & 100,0 & 0,099 \\
\hline Sim & 79 & 55,2 & 64 & 44,8 & 143 & 100,0 & \\
\hline \multicolumn{8}{|l|}{ Fumo: } \\
\hline Não & 385 & 62,1 & 235 & 37,9 & 620 & 100,0 & 0,110 \\
\hline Sim & 27 & 50,9 & 26 & 49,1 & 53 & 100,0 & \\
\hline \multicolumn{8}{|l|}{ Café: } \\
\hline Não & 217 & 66,0 & 112 & 34,0 & 329 & 100,0 & 0,014 \\
\hline Sim & 195 & 56,7 & 149 & 43,3 & 344 & 100,0 & \\
\hline \multicolumn{8}{|l|}{ Tererê: } \\
\hline Não & 164 & 58,2 & 118 & 41,8 & 282 & 100,0 & 0,166 \\
\hline Sim & 248 & 63,4 & 143 & 36,6 & 391 & 100,0 & \\
\hline \multicolumn{8}{|c|}{ Chimarrão: } \\
\hline Não & 369 & 61,8 & 228 & 38,2 & 597 & 100,0 & 0,331 \\
\hline Sim & 42 & 56,0 & 33 & 44,0 & 75 & 100,0 & \\
\hline \multicolumn{8}{|c|}{ Atividade física: } \\
\hline Não & 192 & 57,7 & 141 & 42,3 & 333 & 100,0 & 0,056 \\
\hline Sim & 223 & 64,8 & 121 & 35,2 & 344 & 100,0 & \\
\hline
\end{tabular}

Legenda: Questionário de Berlim - QB.

Fonte: Gaudioso et al. (2020).

Como anteriormente, cabe ressaltar que a prática de atividade física tende a se associar a uma menor proporção de alteração do sono, muito embora não tenha atingido o nível de significância aqui adotado. Já demais variáveis deste grupo não influenciaram o resultado do QB.

O resultado do PSQI o que é identificado pela detecção de qualidade do sono ruim, mostra que o sono ruim ocorre em maior proporção entre as mulheres. Igualmente apresenta maior proporção entre aqueles que têm escolaridade até o ensino médio, já que os demais grupos dessa variável mostram proporção semelhante $(p=0,541)$. O resultado sono ruim está presente em menor proporção entre os católicos, atingindo proporção superior e semelhante entre os que professam as demais religiões consideradas $(p=0,676)$.

Em relação a cor, como ocorre com a variável QB, é ruim em menor proporção entre os que se declaram amarelos, tendo proporção semelhante entre os demais grupos identificados pela sua cor $(p=0,118)$.

A comparação das distribuições da idade entre os que têm sono com qualidade boa ou ruim é semelhante conforme o teste de Mann-Whitney ( $p=0,244$ ), com médias de 33,1 anos para qualidade boa e 32,1 anos para qualidade ruim, corroborando o resultado mostrado para a mesma abordagem com base nas faixas etárias. Existe tendência a uma maior proporção de sono ruim os que não ingerem bebida alcoólica.

A análise multivariada foi realizada, tomando cada uma das variáveis dependentes (QB ou PSQI) em função das variáveis independentes que a elas se associaram significativamente, por um modelo de regressão linear múltipla escalonada ou por etapas (stepwise multiple, linear regression), como já informado anteriormente. Tal método foi aplicado com o objetivo de buscar estabelecer, em cada caso, a ordem de importância e prioridade das variáveis independentes na sua determinação, aferida pelo coeficiente de determinação, expresso em porcentagem da variância do QB ou PSQI.

A proporção de alteração do sono identificada pelo questionário QB aumenta com o aumento da idade, diminui com a melhor QV, é maior entre os declarados brancos do que entre os declarados amarelos, diminui com o nível de escolaridade e é maior entre os que têm o hábito de ingerir café. 
As cinco variáveis independentes respondem, no seu conjunto, por um peso de $10,7 \%$ na determinação da alteração do sono.

\section{Discussão}

A maioria dos entrevistados nesta pesquisa foram mulheres (51,6\%). Dessa forma, o resultado apresentou a qualidade do sono ruim aferido pelo PSQI (60\% das entrevistadas) e alteração do sono detectado pelo QB (38,9\% das entrevistadas). Estudos que corroboram foi realizado com indivíduos da zona rural do município de Pelotas, no Sul do Brasil, seus resultados apresentaram piores médias entre as mulheres. Nesse sentido, fatores hormonais, comportamentais e psicológicos podem ser os responsáveis por essa associação (Machado et al., 2018).

Em mulheres mais jovens, fatores biológicos como a maternidade podem afetar negativamente o sono, enquanto em idades mais avançadas, a menopausa pode promover piora na sua qualidade; a ansiedade e a depressão, conhecidos por serem mais frequentes no sexo feminino, podem estar também presentes nessa relação (Stranges et al., 2012).

Em outro estudo, também com população rural, na cidade de Cássia dos Coqueiros, SP, aponta uma análise sobre a síndrome das pernas inquietas e com isso identificaram essa síndrome mais em mulheres do que em homens (Eckeli et al., 2011). No estudo com 743 adultos, $46,7 \%$ dos indivíduos apresentaram alterações do sono, dentre esses, predominantemente as mulheres (Lopes et al., 2015).

Em 2005, 271 mulheres relataram dormir bem, 67\% dormem de 6 a 8 horas por dia, entretanto, $81 \%$ disseram ter interrupções do sono. Dessas $50 \%$ acordavam até duas vezes por noite, sobre apneia obstrutiva do sono, $26 \%$ tinham características que podem predizer apneia (Souza et al., 2005). Entretanto, contrariam pesquisas com populações rurais que apresentam predominância do sexo masculino, em estudos sobre QV com agricultores orgânicos do interior Paraibano, uma maioria de homens (Pessoa \& Alchieri, 2014). Outro estudo avaliou saúde mental e QV em trabalhadores rurais de uma usina de álcool e açúcar do estado do Mato Grosso do Sul, todos do sexo masculino (Faker, 2009). Também em outra pesquisa em assentamentos foram encontrados 53,16\% homens (Costa Neto, 2016), e em um estudo para avaliar a QV e saúde de trabalhadores pantaneiros detectaram 87,1\% homens (Guimarães, Cerchiari et al., 2018; Guimarães, Meneghel et al. 2018).

Divergindo do estudo com uma população rural de Minas Gerais em 2015, foram avaliadas 587 pessoas entre homens e mulheres, proporções semelhantes a este trabalho, foi possível identificar 37\% dos entrevistados com transtorno do sono, a maioria eram homens (Paula, 2015).

Observa-se que a média de idade dos avaliados nesta pesquisa foi de 32,6 anos, sobretudo, observa-se um aumento significativo da proporção de alteração do sono com o aumento da faixa etária, 36,7 anos no grupo com alteração (p<0,001). A literatura tem intensificado estudos epidemiológicos na população idosa, como por exemplo uma pesquisa realizada na China com 2.416 questionários válidos aplicados em idosos que moram nas regiões rurais, onde foi detectado que 49,7\% dormem mal. Problemas como doenças crônicas, falta de nutrientes na alimentação e a própria idade foram resultantes para um número dessa proporção. Outro estudo foi conduzido entre 266 idosos selecionados aleatoriamente em um subdistrito na província rural de Chiang Rai, norte da Tailândia, aproximadamente 44,0\% dos participantes apresentaram má qualidade do sono (Thichumpa, 2018).

Muito embora, detectar transtornos do sono são mais comuns em estudos com idosos, a literatura tem apresentado dados significativos com a população mais jovem (Cardoso et al., 2009; Vasconcelos et al. 2013; Kang \& Chen, 2009). Este estudo revela que $84 \%$ dos entrevistados tinham entre 15 e 49 anos, uma classificação jovem que veio fortalecer os poucos estudos existentes nesta população pantaneira.

Neste trabalho, quando associado com aumento do nível de escolaridade, é diminuído o risco de alterações do sono, 
reforçando outros experimentos em países de média e baixa renda, ter maior nível educacional diminuiu em $6 \%$ o risco de queixar-se de problemas de sono (Mazzotti et al., 2012); já na China, apresentar menor escolaridade diminuiu em 27\% o risco de ronco e apneia (Liu et al., 2014). Ratifica dessa forma que a escolaridade mais desmedida as atividades ocupacionais manuais e com longas jornadas de trabalho, pode contribuir para o aumento da ocorrência de desordens do sono. Além disso, maior nível educacional está associado a comportamentos benéficos à saúde, prevenindo assim eventos que possam causar problemas no sono (Zanuto et al., 2015).

Entretanto, existem estudos que não encontraram relações (Stranges et al., 2012; Chiu et al., 2012).

A prática de atividade física tende a se associar a uma menor proporção de alteração do sono, muito embora não tenha atingido o nível de significância aqui adotado. As demais variáveis deste grupo não influenciaram o resultado do QB. Entretanto, em estudo que avaliou um grupo com 743 indivíduos, constatou que pessoas com maior escolaridade e praticantes de atividade ou exercício físico apresentam menor ocorrência de transtornos do sono, dos 6.261 estudantes de 14 a 19 anos, foi constatado que aqueles praticantes de exercícios físicos têm menos chances de terem uma percepção negativa da qualidade de sono, se combinado com uma vida fisicamente ativa as chances diminuem mais em comparação aqueles não praticantes e de vida não fisicamente ativa, embora apenas ser fisicamente ativo não reduz as chances de ter uma percepção negativa da qualidade do sono (Lopes et al., 2015). Portanto, os exercícios podem atuar no tratamento e prevenção de alguns distúrbios do ciclo sono-vigília, seja diretamente, por diminuir a divisão do sono, provocar aumento no sono de ondas lentas e diminuição da latência para o sono, ou indiretamente através do controle de peso e aquisição de hábitos saudáveis (Martins et al., 2001; Antunes et al., 2008).

\section{Conclusão}

Este trabalho teve como objetivo avaliar os padrões do sono e seus distúrbios na população pantaneira, como já fora dito, este trabalho é pioneiro na área de estudo e nesta população, os resultados apontaram um aumento significativo da proporção de alteração do sono com o aumento da faixa etária, entretanto, acontece o contrário quanto ao efeito do nível de escolaridade, isto é, diminui a proporção de alteração do sono com o aumento do nível de escolaridade.

Dados também apontam que tanto o estado civil quanto a inatividade (aposentado) e uso de álcool não atingiram um nível de significância. Outra observação importante, foi relacionada ao sexo feminino, que apresentou maiores alterações do sono. Nas mulheres, a diminuição do nível de escolaridade, o aumento da idade e o aumento do número de filhos são fatores que influenciam e colaboram com esse resultado, bem como entre as que têm escolaridade até o ensino médio.

Por fim, o sono é uma condição relevante que abrangem respostas da população principalmente ao alcance dos aspectos gerais de saúde geral desde a caracterização dos moradores dessa região, até a descoberta da prevalência dos acometidos pela má qualidade do sono. Dessa forma, a partir deste trabalho, outros já estão sendo preparados para minimizar os problemas encontrados. Então, é necessário a propagação e difusão desses estudos, assim como pela aproximação do homem do campo com a atenção integral a sua saúde devem ser contínuas, permitindo assim a superação de problemas que inter-relacionam com a $\mathrm{QV}$ do pantaneiro.

\section{Referências}

Abdon, M. M., Silva, J. S. V., Souza, Í. M, Romon, V. T., Rampazzo, J., \& Ferrari, D. L. (2007). Desmatamento no bioma Pantanal até o ano 2002: Relações com a fitofisionomia e limites municipais. Revista Brasileira de Cartografia, 59(1), 17-24. http://mtc-m16b.sid.inpe.br/attachment.cgi/sid.inpe.br/mtcm17@80/2007/06.28.16.16/doc/abdon_desmatamento.pdf

Antunes, H. K. M., Andersen, M. L., Tufik, S., \& Mello, M. T. (2008). Privação de sono e exercício físico. Revista Brasileira de Medicina do Esporte, 14(1), 51-6. http://www.scielo.br/pdf/rbme/v14n1/a10v14n1.pdf 
Bertolazi, A. N. (2008). Tradução, adaptação cultural e validação de dois instrumentos de avaliação do sono: Escala de Sonolência de Epworth e Índice de Qualidade de Sono de Pittsburgh [Dissertação de Mestrado, Universidade Federal do Rio Grande do Sul, Faculdade de Medicina; Porto Alegre. https://www.lume.ufrgs.br/bitstream/handle/10183/14041/000653543.pdf

Cardoso, H. C., Bueno, F. C. C., Mata, J. C., Alves, A. P. R., Jochims, I., Vaz Filho, I. H. R, \& Hanna, M. M. (2009). Avaliação da qualidade do sono em estudantes de Medicina. Revista Brasileira de Educação Médica, 33(3), 349-355. http://www.scielo.br/pdf/rbem/v33n3/05.pdf

Chiu, H. F., Xiang, Y. T., Dai, J., Chan, S. S., Leung, T., Yu, X., Hou, Z.-J., Ungvari, G. S., \& Caine, E. D. (2012). The prevalence of sleep problems and their socio-demographic and clinical correlates in young Chinese rural residents. Psychiatry Research, 200(2-3), 789-94.

Costa Neto, M. C. (2016). Cuidado psicossocial em saúde mental: Estudo em assentamentos rurais do Rio Grande do Norte [Dissertação de Mestrado, Universidade Federal do Rio Grande do Norte, Natal]. https://repositorio.ufrn.br/jspui/bitstream/12345 6789/20903/1/MauricioCiriloDaCostaNeto_DISSERT.pdf

Coxim (Cidade). (2015). Dados do município. Coxim: Prefeitura Municipal. http://www.coxim.ms.gov.br/institucional/dados-do-municipio/13

Delamo, L. M. P. R., Eddine, E. A. C., \& Cunha, S. C. (2010). Memória e constituição do sujeito que vive na região do Pantanal: Atividade e educação em memorialistas pantaneiros. EntreLetras, 3(2), 105-21. https://sistemas.uft.edu.br/periodicos/index.php/entreletras/article/download/957/509/

Eckeli, A. L., Gitaí, L. L., Dach, F., Ceretta, H., Sander, H. H., Passos, A. D. C., Prado, G. F., \& Fernandes, R. M. F. (2011). Prevalence of restless legs syndrome in the rural town of Cassia dos Coqueiros in Brazil. Sleep Medicine, 12(8), 762-7.

Faker, J. N. (2009). A cana nossa de cada dia: saúde mental e qualidade de vida em trabalhadores rurais de uma usina de álcool e açúcar de Mato Grosso do Sul [Dissertação de Mestrado, Universidade Católica Dom Bosco, Campo Grande, MS]. https://site.ucdb.br/public/md-dissertacoes/8075-a-cana-nossa-decada-dia-saude-mental-e-qualidade-de-vida-em-trabalhadores-rurais-de-uma-usina-de-alcool-e-acucar-de-mato-grosso-do-sul.pdf

Ferreira, L. R. C., \& Martino, M. M. F. (2012). Padrão de sono e sonolência do trabalhador estudante de enfermagem. Revista da Escola de Enfermagem da USP, 46, 1.178-83. http://www.scielo.br/pdf/reeusp/v46n5/20.pdf

Finocchio, A. L. F. 1998. O processo de constituição da identidade: As apreensões e mediações sociais e o ato educativo - Um estudo do Paiaguás no Pantanal mato-grossense [Dissertação de Mestrado, Universidade Federal de Mato Grosso do Sul, Campo Grande, MS].

Gaudioso, C. E. V., Magna, L. A., \& Souza, J. C. (2020). Hábitos e distúrbios do sono e sua relação com a qualidade de vida na população do Pantanal. Research, Society and Development, 9(9), e154997123. https://rsdjournal.org/index.php/rsd/article/view/7123/6282

Ghiciuc, C. M., Dima-Cozma, L. C., Bercea, R. M., Lupusoru, C. E., Mihaescu, T., Cozma, S., \& Patacchiolie, F. R. (2016). Desequilíbrio na proporção salivar diurna de testosterona/cortisol em homens com apneia obstrutiva do sono grave: Um estudo observacional. Brazilian Journal of Otorhinolaryngology, 82(5), 529-35. http://www.scielo.br/pdf/bjorl/v82n5/pt_1808-8694-bjorl-82-05-00529.pdf

Godnho, M. R., Ferreira, A. P., Santos, A. S. P., \& Rocha, F. S. A. (2017). Fatores associados à qualidade do sono dos trabalhadores técnico-administrativos em educação de uma universidade pública. Revista de Medicina e Saúde de Brasília, 6, 303-20. https://portalrevistas.ucb.br/index.php/rmsbr/article/download/8624/5482

Guimarães, L. A. M., Cerchiari, E. A. N., Meneghel, V., Minari, M. R. T., Massuda Junior, J., \& Tutya, S. T. B. (2018). Saúde mental do trabalhador do Pantanal Sul-Mato-Grossense, Brasil. In LAM Guimarães, \& EAN Cerchiari (Org.), Saúde do trabalhador do pantanal de Aquidauana, MS, Brasil [pp. 6182]. Campo Grande, MS: UCDB.

Guimarães, L. A. M., Meneghel, V., Fontoura Junior, E. E., Massuda Junior, J., Gomes, E. C. V. V., \& Oliveira, F. F. (2018). Qualidade de vida e aspectos de saúde em trabalhadores pantaneiros. Arquivos Brasileiros de Psicologia, 70(2), 141-57. http://pepsic.bvsalud.org/pdf/arbp/v70n2/11.pdf

Harris, M. B., Arcangelo, C., Pinto, E. C. T., Camargo, G., Ramos Neto, M. B., \& Silva, S. M. (2005). Estimativas de perda da área natural da Bacia do Alto Paraguai e Pantanal Brasileiro. Campo Grande, MS: Conservação Internacional. [Relatório técnico não publicado]. http://riosvivos.org.br/arquivos/1783901110.pdf

Instituto Brasileiro de Geografia e Estatística. (2019). Coxim. IBGE. https://cidades.ibge.gov.br/brasil/ms/coxim/panorama

Kang, J. H., \& Chen, S. C. (2009). Effects of an irregular bedtime schedule on sleep quality, daytime sleepiness, and fatigue among university students in Taiwan. BMC Public Health, 9, 248. https://bmcpublichealth.biomedcentral.com/track/pdf/10.1186/1471-2458-9-248

Liu, J., Wei, C., Huang, L., Wang, W., Liang, D., Lei, Z., Wang, F., Wang, X., Hou, X., \& Tang, X. (2014). Prevalence of signs and symptoms suggestive of obstructive sleep apnea syndrome in Guangxi, China. Sleep and Breathing, 18(2), 375-82.

Lopes, J. M., Gomes, F. S. G., Galvão, D. F., \& Medeiros, J. L. A. (2015). Associação da depressão com as características sociodemográficas, qualidade do sono e hábitos de vida em idosos do Nordeste brasileiro: estudo seccional de base populacional. Revista Brasileira de Geriatria e Gerontologia, 18(3), 521-31. http://www.scielo.br/pdf/rbgg/v18n3/1809-9823-rbgg-18-03-00521.pdf

Machado, A. K. F., Wendt, A., Wehrmeister, F. C. (2018). Problemas de sono e fatores associados em população rural de um município no Sul do Brasil. Revista de Saúde Pública, 52 (Supl 1), 5s. http://www.scielo.br/pdf/rsp/v52s1/pt_0034-8910-rsp-52-s1-S1518-52-87872018052000260.pdf

Martins, P. J. F., Mello, M. T., \& Tufik, S. (2001). Exercício e sono. Revista Brasileira de Medicina do Esporte, 7(1), 28-36. http://www.scielo.br/p df/rbme/v7n1/v7n1a06.pdf

Mazzotti, D. R., Guindalini, C., Sosa, A. L., Ferri, C. P., \& Tufik, S. (2012). Prevalence and correlates for sleep complaints in older adults in low and middle income countries: A 10/66 Dementia Research Group study. Sleep Medicine, 13(6), 697-702. 
Mioto, C. L., Paranhos Filho, A. C., \& Albrez, E. A. (2012). Contribuição à caracterização das sub-regiões do Pantanal. Entre-Lugar; 3(6), 165-80. http://ojs.ufgd.edu.br/index.php/entre-lugar/article/download/2453/1404

Neves, G. S. M. L., Giorelli, A. S., Florido, P., \& Gomes, M. M. (2013). Transtornos do sono: Visão geral. Revista Brasileira de Neurologia, 49, 57-71. http://files.bvs.br/upload/S/0101-8469/2013/v49n2/a3749.pdf

Nogueira, A. X. (1990). O que é Pantanal. Brasiliense.

Oliveira, C. C. S., Tufik, S., Andersen, M. L., Muniz, D. D., Fernandes-Silva, M. M., \& Silvestre, O. M. (2019). Distúrbios do sono prejudicam a saúde cardiovascular ideal. Arquivos Brasileiros de Cardiologia, 112(6), 807-8. http://www.scielo.br/pdf/abc/v112n6/pt_0066-782X-abc-112-06-0807.pdf

Paula, L. K. G. (2015). Herdabilidade da apneia obstrutiva do sono em uma população rural brasileira [Tese de doutorado em Ciências, Universidade de São Paulo, São Paulo]. https://teses.usp.br/teses/disponiveis/5/5150/tde-11092015-154509/publico/LilianKhellenGomesdePaulaVersaoCorrigida.pdf

Pereira, A. S. et al. (2018). Methodology of cientific research. UFSM Editors. https://repositorio.ufsm.br/bitstream/handle/1/15824/ Lic_Computacao_Metodologia-Pesquisa-Cientifica.pdf

Pessoa, Y. S. R. Q., \& Alchieri, J. C. (2014). Qualidade de vida em agricultores orgânicos familiares no interior Paraibano. Psicologia: Ciência e Profissão, 34(2), 330-43. http://www.scielo.br/pdf/pcp/v34n2/v34n2a06.pdf

Ribeiro, C. R. F., Silva, Y. M. G. P., \& Oliveira, S. M. C. (2014). O impacto da qualidade do sono na formação médica. Revista da Sociedade Brasileira de Clínica Médica, 12, 8-14. Acesso em 24 outubro, em http://www.sbcm.org.br/ojs3/index.php/rsbcm/article/view/45/43

Soares, C. S., \& Almondes, K. M. (2012). Sono e cognição: Implicações da privação do sono para a percepção visual e visuoespacial. Psico, 43, 85-92. http://revistaseletronicas.pucrs.br/ojs/index.php/revistapsico/article/download/11102/7622

Souza, C. L., Aldrighi, J. M., \& Lorenzi Filho, G. (2005). Qualidade do sono em mulheres paulistanas no climatério. Revista da Associação Médica Brasileira, 51(3), 170-6. http://www.scielo.br/pdf/ramb/v51n3/a19v51n3.pdf

Stranges, S., Tigbe, W., Gómez-Olivé, F. X., Thorogood, M., \& Kandala, N. B. (2012). Sleep problems: An emerging global epidemic? Findings from the INDEPTH WHO-SAGE study among more than 40,000 older adults from 8 countries across Africa and Asia. Sleep, 35(8), 1.173-81. https://academic.oup.com/sleep/article-pdf/35/8/1173/26620383/aasm.35.8.1173.pdf

Thichumpa, W., Howteerakul, N., Suwannapong, N., \& Tantrakul, V. (2018). Sleep quality and associated factors among the elderly living in rural Chiang Rai, northern Thailand. Epidemiol Health, 40, e2018018. https://www.e-epih.org/upload/pdf/epih-40-e2018018.pdf

Vasconcelos, H. C. A., Fragoso, L. V. C., Marinho, N. B. P., Araújo, M. F. M., Freitas, R. W. J. F., Zanetti, M. L., \& Damasceno, M. M. C. (2013). Correlação entre indicadores antropométricos e a qualidade do sono de universitários brasileiros. Revista da Escola de Enfermagem da USP, 47(4), 852-9. http://www.scielo.br/pdf/reeusp/v47n4/0080-6234-reeusp-47-4-0852.pdf

Vaz, A. P., Drummond, M., Mota, P. C., Severo, M., Almeida, J., \& Winck, J. C. (2011). Tradução do Questionário de Berlim para língua Portuguesa e sua aplicação na identificação da SAOS numa consulta de patologia respiratória do sono. Revista Portuguesa de Pneumologia, 17(2), 59-65. https://www.sciencedirect.com/science/article/pii/S0873215911700150/pdf?md5=242e3baac6e917862fb8a0f77690fab5\&pid=1-s2.0-S0873215911700150main.pdf

Zanuto, E. A. C., Lima, M. C. S., Araújo, R. G., Silva, E. P., Anzolin, C. C., Araujo, M. Y. C., Codogno, J. S., Christofaro, D. G. D., \& Fernandes, R. A. Distúrbios do sono em adultos de uma cidade do Estado de São Paulo. Rev Bras Epidemiol. 2015; 18(1):42-53. http://www.scielo.br/pdf/rbepid/v18n1/1415790X-rbepid-18-01-00042.pdf 